\title{
The Diameter Space, A Restriction of the Drury-Arveson-Hardy Space
}

\author{
N. Arcozzi, R. Rochberg, and E. Sawyer
}

\section{Introduction and Summary}

We consider Carleson measures, Hankel matrices, and interpolation of values on certain reproducing kernel Hilbert spaces which we call the diameter spaces. We begin by reviewing results for the classical Hardy space which we denote $D A H_{1}$ and its associated diameter space. Our $n$-dimensional analog of the Hardy space is the Drury-Arveson-Hardy space, $D A H_{n}$, a space of holomorphic functions on the unit ball in $\mathbb{C}^{n}$; its associated diameter space, $D_{n}$, is a space of real analytic functions on the unit ball in $\mathbb{R}^{n}$. We will see that, as is true in one dimension, some questions which are difficult for $D A H_{n}$ simplify substantially for $D_{n}$. Thus $D_{n}$ may be a useful starting point for analysis of problems on $D A H_{n}$.

In the next section we recall background and establish notation. In the following section we recall the results for $n=1$ and make some comparisons between $D A H_{1}$ and $D_{2}$. In Section 4 we consider positive definite Hankel operators on these space. We find that the classical Hardy space results extend to $D A H_{n}$ and also extend to Besov-Sobolev potential spaces intermediate between those spaces and Dirichlet spaces.

In the final section we will show how some of the results about interpolating sequences for $D_{n}$ can be localized and the local versions combined to obtain characterizations of some interpolating sequences for $D A H_{n}$ which accumulate to the boundary with controlled geometry.

\section{Background and Notation}

In this section we collect notation and definitions and recall some basic facts. We refer to $[\mathbf{A M}]$ and $[\mathbf{A R S 3}]$ for details.

2.1. RKSH's. By a reproducing kernel Hilbert space, RKHS, we mean the following. We are given a set $X$ and a positive definite function $K(\cdot, \cdot)$, the kernel function, defined on $X \times X$. For each $x \in X$ we introduce the kernel function for $x, K_{x}(\cdot):=K(x, \cdot)$. Define the inner product of two kernel functions by

$$
\left\langle K_{x}, K_{y}\right\rangle=K_{x}(y)=K(x, y)
$$

and extend this to finite linear combinations of kernel functions as a sesquilinear form. In particular $\left\|K_{x}\right\|^{2}=\left\langle K_{x}, K_{x}\right\rangle=K(x, x)$. Because $K$ is positive definite this inner product is positive definite and thus we have a pre-Hilbert space. The 
completion, $\mathcal{H}_{X, K}$, is the RKHS generated by $X$ and $K$. Often one suppresses the dependence on $X$ but we want to distinguish a space of functions on $X$ from a space of functions on $Y, Y \subset X$, even if there is a natural isometric isomorphism of the two function spaces. We keep the distinction because some of the constructs we look at, for instance interpolating sequences, are defined in terms of the pair; the set and the space of functions defined on that set.

If $f$ is a linear combination of kernel functions we have the characteristic reproducing property that for any $x \in X$

$$
f(x)=\left\langle f, K_{x}\right\rangle
$$

and we take this as our definition of $f(x)$ for general $f$. In our particular cases this will clearly agree with the natural definition of $f(x)$.

2.2. Interpolating Sequences, Carleson Measures, Multipliers. It is immediate from (2.1) that $|f(x)| \leq\|f\|\left\|K_{x}\right\|$. Hence given $\left\{z_{j}\right\}=Z \subset X$ the map $\mathcal{I}=\mathcal{I}_{Z}$ of $f \in \mathcal{H}_{X, K}$ to $\mathcal{I} f=\left\{K\left(z_{j}, z_{j}\right)^{-1 / 2} f\left(z_{j}\right)\right\}$ is a map of $\mathcal{H}_{X, K}$ into $\ell^{\infty}(Z)$. If this is a bounded map of $\mathcal{H}_{X, K}$ into and onto $\ell^{2}(Z$, counting measure) then $Z$ is called an interpolating sequence (for $\mathcal{H}_{X, K}$ ).

We say a positive measure $\mu$ on $X$ is a Carleson measure for $\mathcal{H}_{X, K}$ if the natural inclusion of $\mathcal{H}_{X, K}$ into $L^{2}(X, d \mu)$ is continuous; $\exists c>0 \forall h \in \mathcal{H}_{X, K}$

$$
\int_{X}|h(x)|^{2} d \mu(x)=\|h\|_{L^{2}(X, d \mu)}^{2} \leq c\|h\|_{\mathcal{H}_{X, K}}^{2} .
$$

There are two natural necessary conditions that a sequence $\left\{z_{i}\right\}=Z \subset X$ must satisfy to be an interpolating sequence. First, the measure

$$
\mu_{Z}=\sum_{i} K\left(z_{i}, z_{i}\right)^{-1} \delta_{z_{i}} \text { is a Carleson measure. }
$$

This is just a reformulation of the requirement that $\mathcal{I}_{Z}$ be bounded. We denote the normalized kernel functions by $k_{x}:=K(x, x)^{-1 / 2} K_{x}$. A reformulation of $(\mathrm{CM})$ is that the Gramm matrix

$$
G=\left(\left\langle k_{z_{i}}, k_{z_{j}}\right\rangle\right)
$$

must be a bounded matrix. The second condition an interpolating sequence must satisfy is a separation condition which is conveniently described in terms of the metric $d_{\mathcal{H}}$ on $X$ where

$$
d_{\mathcal{H}}(x, y):=\left(1-\left|\left\langle k_{x}, k_{y}\right\rangle\right|^{2}\right)^{1 / 2} .
$$

In order for $Z$ to be an interpolating sequence it must be true that

$$
\exists \varepsilon>0, \forall i, j, i \neq j ; d_{\mathcal{H}}\left(z_{i}, z_{j}\right) \geq \varepsilon .
$$

This is necessary because if $Z$ is an interpolating sequence then, given $i, j, i \neq j$, it must be possible to find it $f \in \mathcal{H}_{X, K}$ so that $f\left(z_{i}\right)=0$ and $f\left(z_{j}\right)=K\left(z_{j}, z_{j}\right)^{1 / 2}$ with uniform control of $\|f\|_{\mathcal{H}_{X, K}}$. This observation together with a small bit of functional analysis shows that (SEP) is necessary.

The multiplier algebra of $\mathcal{H}_{X, K}, \mathcal{M}_{\mathcal{H}}$, is the algebra of functions $f$ on $X$ for which the operator of pointwise multiplication by $f, M_{f}$, is bounded on $\mathcal{H}_{X, K}$. In other words there is a $c>0$ so that for all $h \in \mathcal{H}_{X, K}$

$$
\|f h\|_{\mathcal{H}_{X, K}} \leq c\|h\|_{\mathcal{H}_{X, K}} .
$$


This is a Banach algebra when normed by

$$
\|f\|_{\mathcal{M}_{\mathcal{H}}}=\left\|M_{f}\right\|_{\text {operator }} .
$$

The algebra $\mathcal{M}$ might contain only constants and is always contained in the set of bounded functions on $X$. In particular, given a sequence $\left\{z_{j}\right\}=Z \subset X$ the restriction map taking $f \in \mathcal{M}_{\mathcal{H}}$ to $\left\{f\left(z_{j}\right)\right\}$ takes $\mathcal{M}_{\mathcal{H}}$ into $\ell^{\infty}(Z)$. If that map takes $\mathcal{M}_{\mathcal{H}}$ onto $\ell^{\infty}(Z)$ then $Z$ is called an interpolating sequence for $\mathcal{M}_{\mathcal{H}}$ or a multiplier interpolating sequence.

2.3. The Pick Property. Given $\mathcal{H}_{X, K}$ consider the following interpolation problem for the multiplier algebra $\mathcal{M}_{\mathcal{H}}$.

Problem 1 (Pick). Given $\left\{w_{i}\right\}_{i=1}^{n} \subset \mathbb{C},\left\{\lambda_{i}\right\}_{i=1}^{n} \subset X$, is there $m \in \mathcal{M}_{\mathcal{H}}$ with $\|m\|_{\mathcal{M}_{\mathcal{H}}} \leq 1$ and $m\left(\lambda_{i}\right)=w_{i}, i=1, \ldots, n$ ?

There is a necessary condition for this problem to have an affirmative answer which is rather easy to obtain. If that condition is always sufficient we say that $\mathcal{H}_{X, K}$ has the Pick property.

Definition 1. We say $\mathcal{H}_{X, K}$ has the Pick property if for any data $\left\{w_{i}\right\}_{i=1}^{n} \subset \mathbb{C}$, $\left\{\lambda_{i}\right\}_{i=1}^{n} \subset X$ the condition that the "Pick matrix" is positive semidefinite,

$$
\left[\left(1-w_{i} \bar{w}_{j}\right) K\left(\lambda_{i}, \lambda_{j}\right)\right]_{i, j=1}^{n} \geq \geq 0
$$

is necessary and sufficient for the Pick problem to have an affirmative solution.

It is a reformulation of a classical theorem of Pick that the Hardy space has this property. All the spaces we will consider have the Pick property as can be seen from the criteria in $[\mathbf{A M}]$.

We now record two results related to the Pick property.

THEOREM 1 ([AM, pg. 133]). If $\mathcal{H}_{X, K}$ has the Pick property then a sequence $Z \subset X$ is an interpolating sequence for $\mathcal{H}_{X, K}$ if and only if it is an interpolating sequence for $\mathcal{M}_{\mathcal{H}}$.

The second result concerns spaces which also satisfy an additional technical condition introduced by Böe [B2].

Condition B: Given $\left\{z_{j}\right\}=Z \subset X$, if the Gramm matrix

$$
G=\left(\left\langle k_{z_{i}}, k_{z_{j}}\right\rangle\right)
$$

is a bounded matrix then the matrix with all terms made positive

$$
\operatorname{Pos} G=\left(\left|\left\langle k_{z_{i}}, k_{z_{j}}\right\rangle\right|\right)
$$

is also bounded.

Theorem 2 ([B2] $]$ ). If $\mathcal{H}_{X, K}$ has the Pick property and satisfies Condition B then a necessary and sufficient condition on a sequence $Z$ that it be an interpolating sequence for $\mathcal{H}_{X, K}$ (and hence also for $\mathcal{M}_{\mathcal{H}}$ ) is that it satisfy both (SEP) and (CM).

Böe states his theorem for spaces of holomorphic functions but, as he notes, his proof yields what we just stated. In fact his proof also yields a bit more. 
Theorem 3. Suppose $\mathcal{H}_{X, K}$ has the Pick property. Suppose the sequence $Z \subset$ $X$ satisfies both (SEP) and (CM), and, also, for every subsequence $Z_{\text {sub }} \subset Z$, we have that the matrix

$$
\operatorname{Pos} G_{s u b}=\left(\left|\left\langle k_{z_{i}}, k_{z_{j}}\right\rangle\right|\right)_{z_{i}, z_{j} \in Z_{\text {sub }}}
$$

is bounded with a bound only depending on $Z$. Then $Z_{\text {sub }}$ is an interpolating sequence.

\subsection{Particular Spaces.}

2.4.1. The Drury-Arveson-Hardy Spaces. Let $\mathbb{B}_{\mathbb{C}}^{n}$ be the open unit ball in $\mathbb{C}^{n}$. The Drury-Arveson-Hardy space, $D A H_{n}$, is the RKHS on $X=\mathbb{B}_{\mathbb{C}}^{n}$ with the kernel function

$$
K(w, z)=\frac{1}{1-\langle z, w\rangle}=\frac{1}{1-\sum z_{i} \bar{w}_{i}} .
$$

(Thus $\langle z, w\rangle$ is the Hermitian inner product on $\mathbb{C}^{n}$, linear in the first variable, conjugate linear in the second.) See $[\mathbf{A}],[\mathbf{A M}],[\mathbf{C h}]$, and $[\mathbf{A R S 3}]$ for more information about this space.

Alternatively $D A H_{n}$ is the Hilbert space of holomorphic functions in $\mathbb{B}_{\mathbb{C}}^{n}, f=$ $\sum a_{\alpha} z^{\alpha}$, in which the monomials are an orthogonal basis and have norms given by

$$
\left\|z^{\alpha}\right\|^{2}=\frac{\alpha !}{|\alpha| !}
$$

This space has an alternative description as a Besov-Sobolev potential space, it is the space of holomorphic functions on the ball for which

$$
\int_{\mathbb{B}_{\mathbb{C}}^{n}}\left\|R^{m} f(z)\right\|^{2}\left(1-|z|^{2}\right)^{2 m-n} d V(z)<\infty .
$$

Here $R$ is the radial differentiation operator $m$ is an integer with $2 m+1>n$ and $d V$ is volume measure. One can construct an equivalent norm for $D A H_{n}$ using this quantity however the Pick property is a isometric property, lost in passing to a space with equivalent norm.

For $n=1$ this is the classical Hardy space; the space of holomorphic functions on the unit disk with reproducing kernel $K(z, w)=(1-z \bar{w})^{-1}$. The monomials $\left\{z^{n}\right\}_{n=0}^{\infty}$ are an orthonormal basis and, identifying a $f$ in $D A H_{1}$ with its boundary values, we have the (exact) norm formula

$$
\|f\|=\left(\frac{1}{2 \pi} \int_{0}^{2 \pi}\left|f\left(e^{i \theta}\right)\right|^{2} d \theta\right)^{1 / 2} .
$$

2.4.2. More Generally. For $0<\sigma \leq 1 / 2$ we also consider $B_{2}^{\sigma}\left(\mathbb{B}_{\mathbb{C}}^{n}\right)$, the HSRK's on $X=\mathbb{B}_{\mathbb{C}}^{n}$ with kernel functions

$$
K_{\sigma}(w, z)=\frac{1}{(1-\langle z, w\rangle)^{2 \sigma}} .
$$

Thus $B_{2}^{1 / 2}\left(\mathbb{B}_{\mathbb{C}}^{n}\right)=D A H_{n}$. In general these are analytic Besov-Sobolev spaces characterized by, for integer $m$ with $2 m+2 \sigma>n$,

$$
\int_{\mathbb{B}_{\mathbb{C}}^{n}}\left\|R^{m} f(z)\right\|^{2}\left(1-|z|^{2}\right)^{2 m+2 \sigma-n-1} d V(z)<\infty .
$$


2.4.3. A Digression for Clarification. We will frequently be in the following situation. We have a set $X$ and its kernel function $K^{X}$ and a subset $Y \subset X$. The kernel $K^{X}$ restricts to a function $K^{Y}$ on $Y \times Y ; K^{Y}\left(y, y^{\prime}\right):=K^{X}\left(y, y^{\prime}\right)$ for $\left(y, y^{\prime}\right) \in Y \times Y$ which is automatically positive definite.

Given this data there various spaces and operators which can be considered. First there are the two RKHS's, $\mathcal{H}_{X, K^{X}}$ and $\mathcal{H}_{Y, K^{Y}}$. There are also natural maps; the extension map, $\mathcal{E}$, defined first on kernel functions by $\mathcal{E} K_{y}^{Y}=K_{y}^{X}$ and extended by linearity, and the restriction map $\mathcal{R}$ taking functions on $X$ to functions on $Y$ by restricting the domain of definition of the function to $Y$. There are two function spaces of interest defined on $Y ; \mathcal{H}_{Y, K^{Y}}$ and the space of restrictions, $\mathcal{R}\left(\mathcal{H}_{X, K^{X}}\right)$, normed by the quotient norm

$$
\|f\|_{\mathcal{R}\left(\mathcal{H}_{X, K^{X}}\right)}=\inf \left\{\|g\|_{\mathcal{H}_{X, K_{X}}}: \mathcal{R} g=f\right\}
$$

We will also consider two subspaces of $\mathcal{H}_{X, K^{X}}, \overline{\operatorname{Span}\left\{K_{y}^{X}: y \in Y\right\}}$ and $\left(V_{Y}\right)^{\perp}$ where $V_{Y}$ is the subspace of $\mathcal{H}_{X, K^{X}}$ of functions which vanish on $Y$.

We collect here relations between these objects. We will use them later, often without further mention. Working through the definitions and doing standard functional analysis on Hilbert spaces gives the following:

Proposition 1. (1) $\mathcal{E}$ extends by linearity and continuity to an isometric map of $\mathcal{H}_{Y, K^{Y}}$ onto $\overline{\operatorname{Span}\left\{K_{y}^{X}: y \in Y\right\}}$,

(2) $\mathcal{E}^{*}=\mathcal{R}$,

(3) $\mathcal{E}^{*} \mathcal{E}=\mathcal{R} \mathcal{E}=\mathcal{I}_{\mathcal{H}_{Y, K^{Y}}}$, the identity operator on $\mathcal{H}_{Y, K^{Y}}$,

(4) $\mathcal{R}^{*} \mathcal{R}=\mathcal{E} \mathcal{R}=\mathcal{P}_{\left(V_{Y}\right)^{\perp}}$, the orthogonal projection of $\mathcal{H}_{X, K^{X}}$ onto $\left(V_{Y}\right)^{\perp}$.

(5) $\mathcal{H}_{Y, K^{Y}}=\mathcal{R}\left(\mathcal{H}_{X, K^{X}}\right)$, they are the same spaces of functions with the same norm,

(6) $\overline{\operatorname{Span}\left\{K_{y}^{X}: y \in Y\right\}}=\left(V_{Y}\right)^{\perp}$, they are the same spaces of functions with the same norm,

(7) A positive measure $\mu$ supported on $Y$ is a Carleson measure for $\mathcal{H}_{Y, K^{Y}}$ if and only if it is a Carleson measure for $\mathcal{H}_{X, K^{x}}$, and

(8) If $\mathcal{H}_{X, K^{X}}$ has the Pick property then so does $\mathcal{H}_{Y, K^{Y}}$. In that case a sequence $Z \subset Y$ is an interpolating sequence for $\mathcal{H}_{Y, K^{Y}}$ if and only if it is a multiplier interpolating sequence for $\mathcal{H}_{Y, K^{Y}}$ if and only if it is an interpolating sequence for $\mathcal{H}_{X, K^{X}}$ if and only if it is a multiplier interpolating sequence for $\mathcal{H}_{X, K^{X}}$.

If $V_{Y}=\{0\}$ then $\mathcal{E}$ and $\mathcal{R}$ are isometric isomorphisms between $\mathcal{H}_{Y, K^{Y}}$ and $\mathcal{H}_{X, K^{X}}$. However we will continue to view the two as different function spaces. On the other hand we will encounter situations where we have two HSRK's which are not literally the same but which we shall discuss as if they were. We will do this for $\mathcal{H}_{X, K}$ and $\mathcal{H}_{X^{\prime}, K^{\prime}}$ if there is a natural identification of $X$ with all of $X^{\prime}$ which carries $K$ to $K^{\prime}$. For example we do this with the HSRK defined by

$$
\begin{aligned}
X & =\{x \in \mathbb{R}:-1<x<1\} \\
K(x, y) & =(1-x y)^{-1}
\end{aligned}
$$


and the one defined by

$$
\begin{aligned}
X^{\prime} & =\{x+i 0 \in \mathbb{C}:-1<x<1\} \\
K^{\prime}(x+i 0, y+i 0) & =(1-x y)^{-1} .
\end{aligned}
$$

2.4.4. The Diameter Spaces. Let $\mathbb{B}_{\mathbb{R}}^{n}$ be the open unit ball in $\mathbb{R}^{n}$. The diameter space, $D_{n}$, is the RKHS on $X=\mathbb{B}_{\mathbb{R}}^{n}$ with the kernel function given by, for $u, v \in \mathbb{B}_{\mathbb{R}}^{n}$,

$$
L(u, v)=\frac{1}{1-(u, v)}=\frac{1}{1-\sum u_{i} v_{i}} .
$$

(Here $(u, v)$ is the real inner product on $\mathbb{R}^{n}$.) The reproducing kernels for the points of the ball are $L_{u}(\cdot)=L(u, \cdot)$.

We can identify $\mathbb{B}_{\mathbb{R}}^{1}$ with the horizontal diameter $\mathbf{D} \subset \mathbb{B}_{\mathbb{C}}^{1}$ and, as mentioned earlier, identify $D_{1}$ with the HSRK on $\mathbf{D}$ with kernel function obtained by restricting $K(z, w)=(1-z \bar{w})^{-1}$ to $\mathbf{D} \times \mathbf{D}$. In the notation of Proposition $1 V_{Y}$, the subspace of functions in the Hardy space which vanish on $\mathbf{D}$, consists of the zero function. Hence the maps $\mathcal{R}$ and $\mathcal{E}$ of Proposition 1 give isometric isomorphisms of $D A H_{1}$ and $D_{1}$.

The situation is similar for general $n$ if we regard $\mathbb{B}_{\mathbb{R}}^{n}$ as sitting inside $\mathbb{B}_{\mathbb{C}}^{n}$ as the "diameter":

$$
\mathbf{D}^{n}=\left\{\left(x_{1}, \ldots, x_{n}\right) \in \mathbb{B}_{\mathbb{C}}^{n}: x_{i} \in \mathbb{R}, i=1, \ldots n\right\} .
$$

Again $V_{Y}=\{0\}$, now because a holomorphic function on $\mathbb{B}_{\mathbb{C}}^{n}$ which vanishes on $\mathbf{D}^{n}$ must vanish identically as can be seen, for instance, by noting that for holomorphic functions $f$ we have

$$
\frac{\partial^{\alpha}}{\partial z^{\alpha}} f=\frac{\partial^{\alpha}}{\partial x^{\alpha}} f
$$

The maps of Proposition 1 now give isometric isomorphisms of $D_{n}$ and $D A H_{n}$.

$$
\text { 3. } D A H_{1}, D_{1}, D_{2}
$$

We begin by introducing two decompositions and associated tree structures on the unit disk $\mathbb{B}_{\mathbb{C}}^{1}$. The first is the standard decomposition into "top halves of Carleson boxes", the second is a variation of that decomposition. This is more structure than is needed at the moment but it will be useful a bit later.

First a familiar structure. We define a set of boxes on $\mathbb{B}_{\mathbb{C}}^{1}$ by splitting the disk at radii $r_{n}=1-2^{-n}$ and splitting each ring $\left\{r_{n}<|z| \leq r_{n+1}\right\}$ into $2^{n}$ congruent boxes with radial cuts. The tree structure, $\mathcal{T}$, on this set of boxes is described by declaring that $\alpha$ is a successor of $\beta, \alpha \succ \beta$, if the radius through the center of $\alpha$ cuts $\beta$. Note that the successor sets, $S(\alpha)=\cup_{\beta \succeq \alpha} \beta$, are approximately squares of dimension $2^{-n} \times 2^{-n}$, roughly comparable to the superlevel sets of the reproducing kernels for $D A H_{1}$. For the second decomposition we start by splitting the disk into the same rings and again divide each ring into congruent boxes with radial cuts, but now the number of boxes in that ring is to be $\left[2^{n / 2}\right]$. Again the tree structure is described by declaring that $\alpha$ is a successor of $\beta$ if the radius through the center of $\alpha$ cuts $\beta$. Thus the successor sets $S(\alpha)=\cup_{\beta \succeq \alpha} \beta$ are approximately rectangles of dimension $2^{-n} \times 2^{-n / 2}$, roughly comparable to the superlevel sets of the reproducing kernels for diameter space $D_{2}$. Also note that the number of descendents of a vertex after $n$ generations is quite different for the two trees; in the terminology of [ARS2] $\mathcal{T}$ has tree dimension 1 and $\mathcal{F}$ has tree dimension $1 / 2$. 
Will regard measures on $\mathbb{B}_{\mathbb{C}}^{1}$ as measures on $\mathcal{T}$ or $\mathcal{F}$ by assigning to each tree element the measure of that subset of the disk. We identify measures on a tree with functions on the tree.

For any function on a tree $\mathcal{Q}$ we set

$$
\begin{aligned}
I_{\mathcal{Q}} f(\alpha) & =\sum_{\beta \preceq \alpha} f(\beta), \\
I_{\mathcal{Q}}^{*}(g)(\alpha) & =\sum_{\beta \succeq \alpha} g(\beta) .
\end{aligned}
$$

For measures on a tree $\mathcal{Q}$ we will be interested in the "discrete tree condition": $\exists c>0 \forall \alpha \in \mathcal{Q}$

$$
\sum_{\beta \succeq \alpha} 2^{d(\beta)} I_{\mathcal{Q}}^{*} \mu(\beta)^{2} \leq C I_{\mathcal{Q}}^{*} \mu(\alpha)
$$

Here $d(\beta)$ denotes the number of vertices on the path connecting $\beta$ to the root, $o$, the cell containing the origin. We will also consider a simpler condition, the "simple condition": $\exists c>0 \forall \alpha \in \mathcal{Q}$

(Simple)

$$
2^{d(\alpha)} I_{\mathcal{Q}}^{*} \mu(\alpha) \leq C
$$

By using the sum in (Tree) to dominate the single term with $\beta=\alpha$ we see that (Tree) implies (Simple). The fact that the two conditions are not equivalent is seen by considering certain Cantor-like measures.

3.1. The Hardy Space. We recall several classical results for the Hardy space. Proofs are in $[\mathbf{A M}]$ and $[\mathbf{N}]$.

THEOREM 4. A positive measure $\mu$ on $\mathbb{B}_{\mathbb{C}}^{1}$ is a Carleson measure for the Hardy space $\mathrm{DAH}_{1}$ if and only if it satisfies the condition (Simple) for the tree $\mathcal{T}$.

Note: Here and on later occasions we oversimplify slightly, we really need the condition satisfied for $\mathcal{T}$ and all of its rotations. However, it is easy to see that if the simple condition holds for $\mathcal{T}$ then it also holds uniformly for all rotations of $\mathcal{T}$. Moreover, the discrete tree condition also holds uniformly for all rotations of $\mathcal{T}$ if it holds for $\mathcal{T}$ - see the bottom of page 44 of [ARS3]. In particular this applies to the fattened tree condition below, which is the restriction of the split tree condition on the DA space to the diameter. However, one possible exeption is the split tree condition itself. We do not know if it holds for all rotations of $\mathcal{T}$ when it holds for $\mathcal{T}$.

THEOREM 5. A sequence $Z \subset \mathbb{B}_{\mathbb{C}}^{1}$ is an interpolating sequence for the Hardy space $D A H_{1}$, and hence also a multiplier interpolating sequence, if and only if it satisfies the conditions (CM) and (SEP).

We say that a matrix $C=\left(c_{i, j}\right)_{i, j=0}^{\infty}$ operating on the sequence space $\ell^{2}=$ $\ell^{2}\left(\mathbb{Z}_{+}\right)$is a Hankel matrix if there is a sequence $\left\{m_{k}\right\}_{k=0}^{\infty}$ such that $\forall i, j \geq 0$ $c_{i, j}=m_{i+j}$. One way to construct such matrices is to regard elements of $\ell^{2}$ as sequences of Taylor coefficients of elements of the Hardy space $D A H_{1}$. Specifically, if $A=\left\{a_{j}\right\} \in \ell^{2}$ then $f_{A}(z)=\sum_{0}^{\infty} a_{j} z^{j}$ will be in $D A H_{1}$. Suppose that $\mu$ is a measure on $\mathbb{B}_{\mathbb{C}}^{1}$. Define the linear map $M$ on $\ell^{2}$ by

$$
\langle M A, B\rangle_{\ell^{2}}=\int_{\mathbb{B}_{\mathbb{C}}^{1}} f_{A}(z) \overline{f_{B}(\bar{z})} d \mu(z)
$$


It is immediate that the matrix $\mathcal{M}$ for $M$ with respect to the standard basis of $\ell^{2}$ is the Hankel matrix whose entries are given by the moments of $\mu$ :

(Moments)

$$
\mathcal{M}=\left(m_{i+j}\right) ; m_{k}=\int_{\mathbb{B}_{\mathbb{C}}^{1}} z^{k} d \mu(z), k=0,1, \ldots
$$

Theorem 6. A Hankel matrix $\left(m_{i+j}\right)$ is a bounded operator on $\ell^{2}$ if and only if here is a complex measure $\mu$ on $\mathbb{B}_{\mathbb{C}}^{1}$ such that the numbers $m_{k}$ are given by (Moments) and $|\mu|$ is a Carleson measure.

A comment: There is a rich theory of Hankel operators on the Hardy space. On the other hand, little is known about Hankel operators on the other spaces $B_{2}^{\sigma}\left(\mathbb{B}_{\mathbb{C}}^{n}\right)$.

3.2. The Diameter Space $D_{1}$. We view the diameter space $D_{1}$ as a space of functions on the diameter $\mathbf{D}$. Hence it is natural to look for Carleson measures supported on $\mathbf{D}$ and interpolating sequences inside $\mathbf{D}$. Because the functions in $D_{1}$ are exactly the restrictions to $\mathbf{D}$ of functions in $D A H_{1}$ the descriptions of both the Carleson measures and the interpolating sequence can be read off from the results for the Hardy space.

The results for general Hankel operators can also be carried from $D A H_{1}$ to $D_{1}$. Of those results the ones which seem most intimately related to $D_{1}$ are those for the positive definite Hankel operators. One reason for this is that if $f \in D_{1}$ then $\bar{f}$ $\in D_{1}$ which leads to a conflation of the theory of linear operators and the theory of bilinear forms; see the comments at the end of Section 4.1.

The results for $D_{1}$ are

Theorem 7. (1) A positive measure $\mu$ on $\mathbf{D}$ is a Carleson measure for $D_{1}$ if and only if there is a $C>0$ so that

$$
\mu((-1,-r] \cup[r, 1)) \leq C(1-r), 0 \leq r<1
$$

(2) A. A Hankel matrix $\left(m_{i+j}\right)$ is positive semidefinite if and only if there is a positive measure $\mu$ supported on $\mathbb{R}$ so that

$$
m_{k}=\int_{\mathbf{D}} x^{k} d \mu(x), k=0,1, \ldots
$$

Furthermore the numbers $\left\{m_{k}\right\}$ are bounded if and only if the support of $\mu$ is contained in $\mathbf{D}$.

B. A positive semidefinite Hankel matrix $\left(m_{i+j}\right)$ is bounded on $\ell^{2}$ if and only if there is a measure $\mu$ on $\mathbf{D}$ so that

$$
m_{k}=\int_{\mathbf{D}} x^{k} d \mu(x), k=0,1, . .
$$

and $\mu$ is a Carleson measure for $D_{1}$.

(3) A sequence $Z \subset \mathbf{D}$ is an interpolating sequence and a multiplier interpolating sequence if and only if it is separated; that is, it satisfies (SEP).

Statement $2 \mathrm{~A}$ is the classical solution to the Hamberger moment problem; 2B is due to Widom.

These results can be obtained from those for the Hardy space but in fact these statements can be proved much more easily. The simple proof of the first two statements given in $[\mathbf{P}]$ (Theorem 1.6) uses the solution of the Hamburger moment problem to produce the measure $\mu$ and uses the boundedness of the Hilbert matrix 
$\left((i+j)^{-1}\right)_{i, j=1}^{\infty}$ to obtain norm estimates. For statement 3 , first of all for $Z \subset \mathbf{D}$ it is not hard to check that if $Z$ satisfies (SEP) then it also satisfies (CM). Hence $Z$ is an interpolating sequences by Böe's result, Theorem 2. Although this approach requires some machinery, it is much more straightforward than obtaining the statement from the corresponding result for the Hardy space. (Alternatively, one could just go back to the proof for the Hardy space given by Shapiro and Shields (Section 9.6 in $[\mathbf{A M}])$ and notice that it simplifies substantially for sequences contained in D.)

Having noted that various results are easier for $D_{1}$ than for $D A H_{1}$ we should point out that some things are more subtle. For instance, the function $f(x)=x$ has norm 1 in the multiplier algebra of $D_{1}$. This is immediate given the equivalence of $D_{1}$ with the Hardy space and the norm formula (2.4) but not at all transparent from the definition of $D_{1}$ as a RKHS.

3.3. The Diameter Space $D_{2}$. In order to have a better feeling for the diameter spaces we will spell out a few details for $D_{2}$. $D_{2}$ is a HSRK on $\mathbb{B}_{\mathbb{R}}^{2}=$ $\left\{(x, y): x^{2}+y^{2}<1\right\}$. Its reproducing kernel is

$$
K\left(\left(x_{1}, y_{1}\right),\left(x_{2}, y_{2}\right)\right)=\frac{1}{1-x_{1} x_{2}-y_{1} y_{2}}=\frac{1}{1-\left(\left(x_{1}, y_{1}\right),\left(x_{2}, y_{2}\right)\right)} .
$$

Let $\mathbf{R}_{\theta}$ be the rotation of $\mathbb{B}_{\mathbb{R}}^{2}$ through an angle of $\theta$ and let $\mathbf{U}_{\theta}$ be the induced map of $D_{2} ; \mathbf{U}_{\theta} f((x, y))=f\left(\mathbf{R}_{\theta}(x, y)\right)$. Using the second formula for the kernel function one checks that

$$
\left\langle\mathbf{U}_{\theta} K_{\left(x_{1}, y_{1}\right)}, \mathbf{U}_{\theta} K_{\left(x_{2}, y_{2}\right)}\right\rangle=\left\langle K_{\left(x_{1}, y_{1}\right)}, K_{\left(x_{2}, y_{2}\right)}\right\rangle
$$

and hence $\mathbf{U}_{\theta}$ extends to a unitary map of $D_{2}$; i.e., $D_{2}$ is rotation invariant.

The maps $\mathcal{R}$ and $\mathcal{E}$ of Proposition 1 establish an isometric isomorphism of $D_{2}$ with $\mathrm{DAH}_{2}$ and from it we see that the monomials $x^{\alpha} y^{\beta}, \alpha, \beta \in \mathbb{Z}_{+}$are a complete orthogonal set in $D_{2}$ with norms

$$
\left\|x^{\alpha} y^{\beta}\right\|^{2}=\frac{\alpha ! \beta !}{(\alpha+\beta) !}=\omega_{\alpha, \beta}
$$

Thus $D_{1}$ consists of real analytic functions on the disk, $f(x, y)=\sum_{\alpha, \beta \geq 0} c_{\alpha \beta} x^{\alpha} y^{\beta}$ with $\|f\|^{2}=\sum\left|c_{\alpha \beta}\right|^{2} \frac{\alpha ! \beta !}{(\alpha+\beta) !}$. That is, the coefficients lie on the weighted sequence space $\ell^{2}\left(\mathbb{Z}_{+}^{2}, \omega\right)$ where $\omega=\left\{\omega_{\alpha, \beta}\right\}$. Although we have no simple description of the norms of general functions we do have the fact that, for scalars $a, b$ and $k \in \mathbb{Z}_{+}$

$$
\left\|(a x+b y)^{k}\right\|^{2}=\left(|a|^{2}+|b|^{2}\right)^{k} .
$$

This follows from the orthogonality of the monomials, the formula for norms of monomials, and the binomial theorem; alternatively one could use an operators $\mathbf{U}_{\theta}$ to reduce $a x+b y$ to a monomial. This brings us to the following

Problem 2. Is there an equivalent norm on $\mathrm{D}_{2}$ which is given by integrating the function and its various derivatives on $\mathbb{B}_{\mathbb{R}}^{2}$ ? Of course there is the analogous question for $D_{n}$ for general $n$, including $n=1$.

When we identify $\mathbb{B}_{\mathbb{R}}^{2}$ with $\mathbb{B}_{\mathbb{C}}^{1}$ we can consider two HSRK's on the disk, $D_{2}$ and the Hardy space $D A H_{1}$, and it is interesting to contrast the two. Both spaces consist of real analytic functions. Both spaces have the Pick property. In both cases the interpolating sequences are characterized by (SEP) and (CM) (Theorem 5 above 
and Theorem 12 below.) The most obvious difference is that all the functions in $D A H_{1}$ are complex analytic, holomorphic. Hence the only functions $f$ with both $f$ and $\bar{f}$ in $D A H_{1}$ are the constants. On the other hand complex conjugation is an isometric involution on $D_{2}$. The two spaces also have very different exact zero sets, that is sets such that there is a function in the space which vanishes exactly on that set. For the Hardy space the exact zero sets other than the full disk are discrete subsets of the disk. Given one of those discrete subsets it remains an exact zero set if a finite number of points are added or removed. However the exact zero sets for $D_{2}$ include, for instance the set $H=\left\{x y=1 / 4: x^{2}+y^{2}<1\right\}$. If one removes finitely many points from $H$ the result is not an exact interpolation set nor are the two invdividual branches.

The two spaces are also associated with two different geometries on the disk. This can be seen in various ways such as looking at modulus of continuity estimates for functions in the two spaces, looking at versions of the Schwarz lemma for the two, or comparing the metrics $d(x, y)=\left(1-\left|\left\langle k_{x}, k_{y}\right\rangle\right|^{2}\right)^{1 / 2}$ for the two. We will take a quick look at this last possibility.

First we consider the Hardy space and look at the balls in the $d_{D A H_{1}}(\cdot, \cdot)$ metric. A ball centered at $z$ is obtained by fixing $c, 0<c<1$, and finding all $w$ with $\left|\left\langle k_{z}, k_{w}\right\rangle\right|^{2} \leq c$. We have

$$
\left|\left\langle k_{z}, k_{w}\right\rangle\right|^{2}=\frac{\left(1-|z|^{2}\right)\left(1-|w|^{2}\right)}{|1-z \bar{w}|^{2}}=1-\left|\frac{z-w}{1-\bar{w} z}\right|^{2}
$$

Hence the balls are described by, for some $c^{\prime}$,

$$
\left|\frac{z-w}{1-\bar{w} z}\right| \leq c^{\prime}
$$

But these are just balls in the hyperbolic geometry of the disk. For fixed $z=$ $1-\varepsilon$ with $\varepsilon$ small and positive this is a Euclidean disk inside $\mathbb{B}_{\mathbb{C}}^{1}$ containing $z$. Its Euclidean radius and its Euclidean distance to $\partial \mathbb{B}_{\mathbb{C}}^{1}$ are both comparable to $\varepsilon$.

The disk with the same center in the $d_{D_{2}}(\cdot, \cdot)$ metric is the set of $(x, y)$ with $\left|\left\langle k_{(1-\varepsilon, 0)}, k_{(x, y)}\right\rangle\right|^{2} \leq c$. This gives

$$
\left|\left\langle k_{z}, k_{w}\right\rangle\right|^{2}=\frac{\left(1-(1-\varepsilon)^{2}\right)\left(1-x^{2}-y^{2}\right)}{(1-(1-\varepsilon) x)^{2}} \leq c .
$$

This is a Euclidean ellipse containing $(1-\varepsilon, 0)$, with horizontal extension roughly $\varepsilon$ and vertical dimension approximately $\sqrt{\varepsilon}$. In particular the eccentricity of the ellipse is unbounded as $\varepsilon$ tends to zero.

This difference in geometries is reflected in various ways in the function theory of the two spaces. It is at the base of the difference between the decompositions of the disk into the trees $\mathcal{T}$ and $\mathcal{F}$ described earlier. The cells of $\mathcal{T}$ have approximately the same size and shape as balls of fixed radius in the $d_{D A H_{1}}(\cdot, \cdot)$ metric, the $\mathcal{F}$ cells have, roughly, the size and shape as $d_{D_{2}}(\cdot, \cdot)$ balls of fixed radius. Hence, for instance, if a sequence in $\mathbb{B}_{\mathbb{C}}^{1}$ is an interpolating sequence for $D A H_{1}$ then (SEP) forces an upper bound on the number of points of the sequence in any cell of $\mathcal{T}$. A similar conclusion holds for interpolating sequences for $D_{2}$ but with the cells of $\mathcal{F}$. The cells of $\mathcal{T}$ are those in which a normalized element of the Hardy space can change value by roughly one unit, the cells of $\mathcal{F}$ play that role for the diameter space. 
We noted earlier that the Carleson measures for the Hardy space are described using $\mathcal{T}$. Those for $D_{2}$ are described using $\mathcal{F}$.

TheOREm 8 ([ARS3, $§ 2.3 .5])$. A positive measure $\mu$ on $\mathbb{B}_{\mathbb{C}}^{1}$ is a Carleson measure for the space $D_{2}$ if and only if it satisfies the discrete tree condition (Tree) with respect to the tree $\mathcal{F}$.

Problem 3. The previous discussion indicates why $\mathcal{F}$ rather than $\mathcal{T}$ might play a central role for $D_{2}$. It is not clear however what type of equally straightforward conditions would suggest that the Carleson measures would be characterized by (Tree) rather than (Simple).

This theorem is obtained in [ARS3] as a specialization of the characterization of the Carleson measures for the case $\sigma=1 / 2, D A H_{2}$, to measures which are supported on the diamater $\mathbf{D}^{2}$. It would be interesting to have a direct proof of the theorem.

\section{Hankel Matrices}

The books $[\mathbf{P}]$ and $[\mathbf{N}]$ are references for this section.

4.1. Positive Hankel Matrices. We now give our analog of the part of the previous theorem which concerned Hankel matrices. Our analog is not complete; we do not give a necessary and sufficient condition in terms of the moment matrix itself for a measure to be supported in the ball. The question of what conditions would insure that is very interesting but we have no contribution to offer; see $[\mathbf{P V}]$ for recent results.

Suppose we are given a real bounded function $\left\{\gamma_{\alpha}\right\}_{\alpha \in \mathbb{Z}_{+}^{n}}$ on the semigroup $\left\{\mathbb{Z}_{+}^{n},+\right\}$ consisting of all nonnegative multi-indices $\alpha=\left(\alpha_{1}, \ldots, \alpha_{n}\right)$ under addition. The sequence $\left\{\gamma_{\alpha}\right\}_{\alpha \in \mathbb{Z}_{+}^{n}}$ is positive definite on $\left\{\mathbb{Z}_{+}^{n},+\right\}$ provided

$$
\sum_{\alpha, \beta \in \mathbb{Z}_{+}^{n}} s_{\alpha} \gamma_{\alpha+\beta} \overline{s_{\beta}} \geq 0
$$

which is the statement that the associated Hankel matrix $\Gamma=\left\{\gamma_{\alpha+\beta}\right\}_{\alpha, \beta}$ is positive definite.

Theorem 9. If the Hankel matrix $\Gamma=\left\{\gamma_{\alpha+\beta}\right\}_{\alpha, \beta}$ is positive definite and has bounded entries then $\left\{\gamma_{\alpha}\right\}_{\alpha \in \mathbb{Z}_{+}^{n}}$ is a moment sequence; there is a positive measure $\mu$ supported on the cube $[-1,1]^{n}$ so that

$$
\gamma_{\alpha}=\int_{\mathbb{R}^{n}} x^{\alpha} d \mu(x), \quad \alpha \in \mathbb{Z}_{+}^{n} .
$$

If, furthermore, the matrix defines a bounded operator on $\ell^{2}\left(\mathbb{Z}_{+}^{n}, \omega\right), \omega(\alpha)=\alpha ! /|\alpha| !$ then $\mu$ is supported on $\overline{\mathbf{D}^{n}} \subset \overline{\mathbb{B}_{\mathbb{C}}^{n}}$ and satisfies the Carleson measure estimate for functions in $\mathrm{DAH}_{n}$

$$
\int_{\overline{\mathbf{D}^{n}}}|f|^{2} d \mu \leq c\|f\|_{D A H_{n}}^{2}
$$

Proof. The existence of the measure with required support is Proposition 4.9 of $[\mathbf{B C R}]$. (That result concerns semigroups with involution. In our case the identity map is the involution.) We regard $\mathbb{R}^{n}$ as sitting inside $\mathbb{C}^{n}$ as

$$
\mathbb{R}^{n} \approx\left\{\left(x_{1}, \ldots, x_{n}\right) \in \mathbb{C}^{n}: x_{1}, \ldots, x_{n} \in \mathbb{R}\right\}
$$


and doing this can regard $\mu$ as a measure on $\mathbb{C}^{n}$.

Let $\mathcal{I}$ be the "identity" map from $D A H_{n}$ to $L^{2}\left(\mathbb{C}^{n}, d \mu\right)$ which is certainly well defined for polynomials. We have

$$
\begin{aligned}
\gamma_{\alpha+\beta} & =\int_{\mathbb{C}^{n}} x^{\alpha+\beta} d \mu(x) \\
& =\int_{[-1,1]^{n}} x^{\alpha+\beta} d \mu(x) \\
& =\int_{[-1,1]^{n}} z^{\alpha} \bar{z}^{\beta} d \mu(z) \\
& =\left\langle\mathcal{I} z^{\alpha}, \mathcal{I} z^{\beta}\right\rangle_{L^{2}\left([-1,1]^{n}, d \mu\right)} .
\end{aligned}
$$

Hence for a finite sequences $A=\left\{a_{\alpha}\right\} \in \ell^{2}\left(\mathbb{Z}_{+}^{n}, \omega\right)$ we have, writing $f_{A}(z)=$ $\sum a_{\beta} z^{\beta}$,

$$
\begin{aligned}
\langle\Gamma[\mu] A, A\rangle_{\ell^{2}} & =\sum_{\alpha, \beta=0}^{\infty} \gamma_{\alpha+\beta} a_{\beta} \overline{a_{\alpha}} \\
& =\sum_{\alpha, \beta=0}^{\infty}\left\langle\mathcal{I} z^{\beta}, \mathcal{I} z^{\alpha}\right\rangle_{L^{2}\left([-1,1]^{n}, d \mu\right)} a_{\beta} \overline{a_{\alpha}} \\
& =\left\langle\mathcal{I} \sum_{\beta=0}^{\infty} a_{\beta} z^{\beta}, \mathcal{I} \sum_{\alpha=0}^{\infty} a_{\alpha} z^{\alpha}\right\rangle_{L^{2}\left([-1,1]^{n}, d \mu\right)} \\
& =\int_{[-1,1]^{n}}\left|f_{a}\right|^{2} d \mu .
\end{aligned}
$$

Combining this with the fact that $\|A\|_{\ell^{2}\left(\mathbb{Z}_{+}^{n}, \omega\right)}=\left\|f_{A}\right\|_{D A H_{n}}$ and that the matrix $\Gamma[\mu]$ is bounded we see that for any polynomial we have.

$$
\int|P(z)|^{2} d \mu(z) \leq C\|P\|_{H_{n}^{2}}^{2}
$$

To finish we want to show that $\mu$, which we already know supported in $[-1,1]^{n}$, is also supported in the closed unit complex ball $\overline{\mathbb{B}_{\mathbb{C}}^{n}}$ and hence, combining the two facts, we actually have $\operatorname{supp} \mu \subset \overline{\mathbf{D}^{n}}$. This follows from an argument on page 20 of Arveson's paper $[\mathbf{A}]$; we give a mild generalization of that argument in the proof of the next theorem.

This proof only uses the defining property of Carleson measures, the estimate (4.3), not a characterization of Carleson measures. We do however have a structural description of those measures. We can split $\mu$ into parts carried by the open ball and the boundary:

$$
\mu=\mu_{\mathbb{B}_{\mathbb{C}}^{n}}+\mu_{\partial \mathbb{B}_{\mathbb{C}}^{n}}
$$

and both must be Carleson measures. For $n=1$ the Carleson measure condition for $\mu_{\mathbb{B}_{C}^{n}}$ is in Theorem 8; a similar description holds for $n>2$ by essentially the same argument as that in Section 2.3.5 of [ARS3]. The Carleson measure condition on $\mu_{\partial \mathbb{B}_{\mathbb{C}}^{n}}$ amounts to a trace inequality for a certain nonisotropic Besov space on 
the boundary $\partial \mathbb{B}_{\mathbb{C}}^{n}$, which can be characterized by either a capacity condition or a nonisotropic variant of the Kerman-Sawyer condition.

The proof we just gave works almost unchanged for the spaces $B_{2}^{\sigma}\left(\mathbb{B}_{\mathbb{C}}^{n}\right), 0<\sigma \leq$ $1 / 2$; the case $\sigma=1 / 2$ being the one just presented. Let $\left\{\omega_{n}^{\sigma}(\alpha)\right\}_{\alpha=\left(\alpha_{1}, \ldots, \alpha_{n}\right) \geq 0}$ be the sequence of positive coefficients such that for $f(z)=\sum_{\alpha \geq 0} a_{\alpha} z^{\alpha}$ holomorphic in $\mathbb{B}_{\mathbb{C}}^{n}$ we have

$$
\|f\|_{B_{2}^{\sigma}}^{2}=\sum_{\alpha \geq 0}\left|a_{\alpha}\right|^{2} \omega_{n}^{\sigma}(\alpha), \quad 0<\sigma \leq \frac{1}{2} .
$$

Let $0<\sigma \leq \frac{1}{2}$. Suppose $\mu$ is a positive measure on $\overline{\mathbf{D}^{n}} \subset \overline{\mathbb{B}_{\mathbb{C}}^{n}}$. Let $\Gamma[\mu]=$ $\left[\gamma_{\alpha+\beta}\right]_{\alpha, \beta \geq 0}$, where

$$
\gamma_{\alpha}=\int_{\overline{\mathbb{B}_{\mathbb{C}}^{n}}} z^{\alpha} d \mu
$$

be its associated Hankel matrix.

TheOREM 10. $\Gamma[\mu]$ is a bounded form on $\ell^{2}\left(\omega_{n}^{\sigma}\right)$, i.e. $\forall\left\{a_{\alpha}\right\},\left\{b_{\beta}\right\} \in \ell^{2}\left(\omega_{n}^{\sigma}\right)$,

$$
\left|\sum_{\alpha, \beta \geq 0} \gamma_{\alpha+\beta} a_{\alpha} \overline{b_{\beta}}\right| \leq C\left\|\left\{a_{\alpha}\right\}\right\|_{\ell^{2}\left(\omega_{n}^{\sigma}\right)}\left\|\left\{b_{\beta}\right\}\right\|_{\ell^{2}\left(\omega_{n}^{\sigma}\right)},
$$

if and only if $\mu$ is supported on $\overline{\mathbf{D}^{n}}$ and is a Carleson measure for the Besov-Sobolev space $B_{2}^{\sigma}$, i.e. $\forall f \in B_{2}^{\sigma}$

$$
\int_{\overline{\mathbb{B}_{\mathbb{C}}^{n}}}|f|^{2} d \mu \leq C\|f\|_{B_{2}^{\sigma}}^{2} .
$$

A description of measures supported in $\mathbf{D}^{n}$ which satisfy (4.5) is given in terms of a tree condition as Theorem 3.1 of [ARS2]. For $n=2$ the condition is $\exists C>0$ $\forall \alpha \in \mathcal{F}$

$$
\sum_{\beta \geq \alpha}\left[2^{\sigma d(\beta)} I^{*} \mu(\beta)\right]^{2} \leq C I^{*} \mu(\alpha)<\infty .
$$

As before the condition for the part of the measure living on the boundary is the condition for the existence of a trace and can be described by capacity condition or a nonisotropic variant of the Kerman-Sawyer condition.

Proof. The proof follows exactly the pattern of the previous one up to the last step. To finish we need to show that if a positive measure on $\mathbb{C}^{n}$ satisfies (4.5) then the measure is supported in $\overline{\mathbb{B}_{\mathbb{C}}^{n}}$. If $U$ is a unitary map of $\mathbb{C}^{n}$ then the map $f(\cdot) \rightarrow f(U \cdot)$ is a unitary map of $B_{2}^{\sigma}$. Using this we reduce to showing that if $\mu$ satisfies (4.5) and $\varepsilon>0$ then

$$
\lambda_{\varepsilon}=\mu\left(\left\{\left(z_{1}, \ldots, z_{n}\right) \in \mathbb{C}^{n}: \operatorname{Re} z_{1} \geq 1+\varepsilon\right\}\right)=0 .
$$

We evaluate (4.5) for the function $f(z)=z_{1}^{n}$ and make the natural estimate. This gives

$$
\lambda_{\varepsilon}(1+\varepsilon)^{2 n} \leq C\left\|z_{1}^{n}\right\|_{B_{2}^{\sigma}}^{2} .
$$

When we write the Taylor series for the kernel function in (2.5) there will be a term $c(n, \sigma) z_{1}^{n} \bar{w}_{1}^{n}$ and we have $c(n, \sigma)=\left\|z_{1}^{n}\right\|_{B_{2}^{\sigma}}^{-2}$. Hence

$$
\lambda_{\varepsilon}(1+\varepsilon)^{2 n} \leq C c(n, \sigma)^{-1}=C \prod_{j=1}^{n} \frac{j}{2 \sigma-1+j} .
$$


The left side has exponential growth in $n$, the right hand side has power growth. A contradiction is avoided by concluding that $\lambda_{\varepsilon}=0$.

The theories of Carleson measures and of Hankel matrices are closely linked. The results we just looked at are extreme instances of this and we should indicate why this comes about. A positive measure $\mu$ is a Carleson measure for, let's say, $B_{2}^{\sigma}$, if there is an estimate: $\forall f, g \in B_{2}^{\sigma}$.

$$
\left|\int_{\mathbb{B}_{\mathbb{C}}^{n}} f(z) \overline{g(z)} d \mu\right| \leq C\|f\|\|g\|
$$

On the other hand the moment sequence of $\mu$ will give rise to a bounded Hankel matrix on the sequence space of coefficients of functions in $B_{2}^{\sigma}$ if there is an estimate: $\forall f, g \in B_{2}^{\sigma}$.

$$
\left|\int_{\mathbb{B}_{\mathbb{C}}^{n}} f(z) g(z) d \mu\right| \leq C\|f\|\|g\| .
$$

Using these descriptions it is easy to check that if $|\mu|$ is a Carleson measure then the associated Hankel matrix is bounded. However information doesn't pass easily in the other direction. However if for every $g \in B_{2}^{\sigma}$ one can find $\tilde{g} \in B_{2}^{\sigma}$ so that $\|\tilde{g}\| \leq C\|g\|$ and $\tilde{g}=\bar{g}$ on supp $\mu$ then (4.7) is a consequence of (4.8). In the cases we have been looking at the fact that the matrix was positive definite together with straightforward growth estimates insured that $\operatorname{supp} \mu \subset \overline{\mathbf{D}^{n}}$. In that case for $g(z)=\sum c_{\alpha} z^{\alpha}$ we can choose $\tilde{g}(z)=\sum \bar{c}_{\alpha} z^{\alpha}$ and obtain the equivalence.

The previous argument centered on a property of $\overline{\mathbf{D}^{n}}$ with respect to the space $B_{2}^{\sigma}$. It is not clear what other types of sets have this property (or one close enough to it to have a similar argument work) and it would be interesting to know more. It is a walk through the definitions to see that interpolating sequences are of sets with the property.

4.2. Trace Class Positive Hankel Matrices. The analysis of trace class (a.k.a nuclear) Hankel matrices acting on $\ell^{2}\left(\mathbb{Z}_{+}\right)$is not straightforward but a description is known [N], similar questions haven't been studied for $D A H_{n}, n>1$. However if the Hankel matrix is positive then the analysis is easy and the answer is elegant. As before suppose $\mu$ is a positive measure on $\overline{\mathbf{D}^{n}} \subset \overline{\mathbb{B}_{\mathbb{C}}^{n}}$. Let $\Gamma[\mu]=\left[\gamma_{\alpha+\beta}\right]_{\alpha, \beta \geq 0}$ be its associated Hankel matrix.

THEOREM 11. $\Gamma[\mu]$ is a trace class operator on $\ell^{2}\left(\omega_{n}^{\sigma}\right)$ if and only if

$$
\int_{\overline{\mathbf{D}^{n}}} K_{\sigma}(z, z) d \mu(z)=\int_{\overline{\mathbf{D}^{n}}}(1-\langle z, w\rangle)^{-2 \sigma} d \mu(z)<\infty .
$$

Proof. This can be checked using the fact that for a positive operator $T$ the trace and the trace class norm are both given by $\sum\left\langle T e_{n}, e_{n}\right\rangle$ where the sum is over any orthonormal basis $\left\{e_{n}\right\}$. If one uses the normalized monomials as a basis and uses the Taylor series for the kernel function to read off the norms then the result follows. Alternatively one can pass to the analogous question for Hankel bilinear forms on RKHS's and use that theory as in Corollary 6.3.2 of [Z]. 


\section{Interpolating Sequences}

5.1. $Z \subset \mathbf{D}^{n}$. In this section we consider interpolating sequences $D A H_{n}$. It would be very interesting to know if every sequence $Z \subset \mathbb{B}_{\mathbb{C}}^{n}$ which satisfied $(\mathrm{CM})$ and (SEP) was an interpolating sequence. That is true for $n=1$ but little is known in general. However if $Z \subset \mathbf{D}^{n}$ then all of the entries in the Gramm matrix, (2.2), are positive and hence Condition B is automatically satisfied.

TheOREM 12. A sequence $Z \subset \mathbf{D}^{n} \subset \mathbb{B}_{\mathbb{C}}^{n}$ is an interpolating sequence for $D A H_{n}$, and hence also for $D_{n}$, if and only if it satisfies the conditions (CM) and (SEP).

We now build on this to describe a more general class of interpolating sequences.

5.2. More General Geometry. In this section we use some of the ideas from Section 2.3.5 of [ARS3]. Suppose $\phi$ is a map of class $C^{2}$ of a smoothly bounded domain $\Omega$ in $\mathbb{R}^{n}$ into $\mathbb{B}_{\mathbb{C}}^{n}$ which takes $\partial \Omega$ into $\partial \mathbb{B}_{\mathbb{C}}^{n}$. Suppose further that at $\partial \Omega$ the map $\phi$ is transverse to $\partial \mathbb{B}_{\mathbb{C}}^{n}$ and is in the complex tangential direction; that is, for $x \in \partial \Omega$ and any tangent vector $\mathbf{T}$ to $\partial \mathbb{B}_{\mathbb{C}}^{n}$ at $\phi(x)$ we have $\left\langle\phi^{\prime} \mathbf{T}, \phi\right\rangle=0$. In this case we will call $\phi(\Omega)$ a complex tangential set. The flat model case for $\phi$ is the inclusion of the real ball into the complex ball as $\mathbf{D}^{n}$ as a totally real submanifold.

Proposition 2. Suppose the sequence $Z$ sits in a complex tangential set $S$ in $\mathbb{B}_{\mathbb{C}}^{n}$. Then for each $\zeta \in \partial \mathbb{B}_{\mathbb{C}}^{n}$ there is a neighborhood, $N_{\zeta}$ so that the subsequence $Z \cap N_{\zeta}$ satisfies Condition B with constant that can be taken to be independent of $\zeta$. Hence if that subsequence satisfies conditions (CM) and (SEP) it will be an interpolating sequence.

Proof. The basic idea is straightforward. The hypotheses on $\phi$ insure that for every $x \in \partial \Omega$ there is a $\delta_{0}(x)>0$ so that for any unit vector $T$ at $x$ pointing into $\Omega$ and all $\delta<\delta_{0}(x)$

$$
1-\langle\phi(x), \phi(x+\delta \mathbf{T})\rangle=\delta^{2} \frac{\left|\phi^{\prime}(x)\right|^{2}}{2}+o\left(\delta^{2}\right),
$$

here by $x+\delta \mathbf{T}$ we mean the point in $\partial \bar{\Omega}$ that is obtained by flowing along $\partial \bar{\Omega}$ from $x$ a distance $\delta$ in the direction of $\mathbf{T}$. This is (2.4) of $[\mathbf{A C}]$. We will use this to show that, locally, the real part of the reproducing kernel dominates the modulus which implies Condition B. Here are the details.

Recall that the reproducing kernel is $k_{w}(z)=K(w, z)=\frac{1}{1-\langle z, w\rangle}$. Thus

$$
\operatorname{Re} \frac{1}{1-\langle\phi(x), \phi(x+\delta \mathbf{T})\rangle}=\operatorname{Re} \frac{1}{\delta^{2} \frac{\left|\phi^{\prime}(x)\right|^{2}}{2}+o\left(\delta^{2}\right)} \approx\left|\frac{1}{1-\langle\phi(x), \phi(x+\delta \mathbf{T})\rangle}\right|
$$

for $\delta>0$ sufficiently small uniformly in $x$. Then $\operatorname{Re} K(w, z) \approx|K(w, z)|$ when $z$ and $w$ lie in a sufficiently small neighbourhood $N_{\varsigma}$ of $\varsigma$. Condition B now follows since the nonnegativity of the Gram matrix $G_{\varsigma}=\left(\left\langle k_{z_{i}}, k_{z_{j}}\right\rangle\right)_{z_{i}, z_{j} \in Z \cap N_{\varsigma}}$ yields

$$
\begin{aligned}
\sum_{z_{i}, z_{j} \in Z \cap N_{\varsigma}}\left|\left\langle k_{z_{i}}, k_{z_{j}}\right\rangle\right| \xi_{i} \xi_{j} & \leq C \sum_{z_{i}, z_{j} \in Z \cap N_{\varsigma}} \operatorname{Re}\left\langle k_{z_{i}}, k_{z_{j}}\right\rangle \xi_{i} \xi_{j} \\
& =C \sum_{z_{i}, z_{j} \in Z \cap N_{\varsigma}}\left\langle k_{z_{i}}, k_{z_{j}}\right\rangle \xi_{i} \xi_{j} \leq C|\xi|^{2}
\end{aligned}
$$

for all real vectors $\xi=\left(\xi_{j}\right)$, provided $G_{\varsigma}$ is bounded. This inequality gives the boundedness of $\operatorname{Pos} G_{\varsigma}$, which is Condition B for $G_{\varsigma}$. 
In particular, if $Z \subset \mathbb{B}_{\mathbb{C}}^{n}$ itself satisfies $(\mathrm{CM})$ and (SEP) then so does every subsequence and with constants that are no larger. In that case the second half of the proposition automatically applies.

TheOREM 13. A sequence $Z \subset \mathbb{B}_{\mathbb{C}}^{n}$ that sits in a complex tangential set is an interpolating sequence for $D A H_{n}$ if and only if it satisfies (CM) and (SEP).

To prove this we need to show that the conclusion of the proposition is enough to insure that we have an interpolating sequence. This is true in some generality and hence we present it as a separate result in the next subsection. Completing that proof will also finish the proof of this theorem.

5.3. Local Implies Global. Suppose $M \subset \mathbb{C}^{n}$ has smooth compact boundary $\partial M$, that $\mathcal{H}$ is a Hilbert space of continuous functions on $M$ with reproducing kernels $J_{x}, x \in M$ and normalized reproducing kernel $j_{x}=\left\|J_{x}\right\|^{-1} J_{x}$. We denote the Euclidean balls in $\mathbb{C}^{n}$ by $B(x, r)$. We suppose:

(1) The algebra of multipliers of $\mathcal{H}, \mathcal{M}_{\mathcal{H}}$, separates points of $M$; i.e., given $x, y \in M$ there is an $m \in M$ with $m(x)=1$ and $m(y)=0$.

(2) There is a function $\gamma(t)$ with $\gamma(t) \rightarrow 0$ as $t \rightarrow 0$ so that if $\mu$ is a Carleson measure for $\mathcal{H}$ with $\|\mu\|=1$ and if $x$ is any point of $\partial M$ then $\mu(B(x, t)) \leq$ $\gamma(t)$.

(3) The function $J_{x}(y)$ is bounded away from the diagonal. Given $\varepsilon>0$ there is $A_{\varepsilon}<\infty$ so that

$$
\sup \left\{\left|J_{x}(y)\right|: d(x, y)>\varepsilon\right\}<A_{\varepsilon} .
$$

We will say that a sequence $X=\left\{x_{i}\right\} \subset M$ is a local interpolating sequence with constants $B, \varepsilon_{X}>0$ if it has no accumulation points in $M$ and if for each $x \in \partial M$ the set $X \cap B\left(x, \varepsilon_{X}\right)$ is an interpolating sequence with constant $B$. Note that if $X^{\prime} \subset X$ then $X^{\prime}$ will also be a local interpolating sequence with the same constants.

REMARK 1. These assumptions certainly allow the case of $\mathcal{H}=D A H_{n}$. The polynomials are multipliers which insures the first condition, it is shown in [ARS3] that the Carleson measures for that space satisfy a version of (Simple) and that is more than enough to insure the second condition and the third is a consequence of elementary geometry. We wanted to emphasize that the theorem below uses almost no specific information about $D A H_{n}$; for instance it certainly does not use the explicity formula for the kernel function or the fact that the kernel has the Pick property.

For $X=\left\{x_{i}\right\} \subset M$ let $\mu_{X}$ be the associated measure

$$
\mu_{X}=\sum_{x_{i} \in X}\left\|J_{x_{i}}\right\|^{-2} \delta_{x_{i}},
$$

We note for later that if $X$ is a local interpolation sequence then $\mu_{X}$ is a finite measure. This is true if the sequence is an interpolating sequence by $(\mathrm{CM})$. The conclusion then follows for $X$ using the definition of local interpolating sequence and the compactness of $\partial M$.

We will use the fact (e.g. [B1], Theorem 1.1) that $X=\left\{x_{i}\right\} \subset M$ is an interpolating sequence if and only if it is a Riesz basis for the closed span of $\left\{j_{x_{i}}\right\}$; 
that is, for scalars $\left\{\alpha_{i}\right\}$

$$
\left\|\sum \alpha_{i} j_{x_{i}}\right\|^{2} \sim \sum\left|\alpha_{i}\right|^{2}
$$

Recall that subspaces $A$ and $B$ are said to be at a positive angle if there is an $\varepsilon>0$ so that for any $a \in A, b \in B$ we have

$$
|\langle a, b\rangle| \leq(1-\varepsilon)\|a\|\|b\|
$$

Basic facts about Hilbert space give the following

Proposition 3. Suppose $\left\{x_{i}\right\}_{i \in I}$ is a Riesz basis for its closed linear span X. Let $I_{\alpha}, \alpha=1,2,3$ be disjoint subsets of $I$ and let $X_{\alpha}$ be the closed linear span of $\left\{x_{i}\right\}_{i \in \alpha}, \alpha=1,2,3$ then

(1) for $\alpha=1,2,3$ the set $\left\{x_{i}\right\}_{i \in \alpha}$ is a Riesz basis for $X_{\alpha}$, and

(2) the spaces $X_{\alpha}$ are pairwise at a positive angle.

Conversely, if 1 and 2 are satisfied then $\left\{x_{i}\right\}_{i \in I_{1} \cup I_{2} \cup I_{3}}$ is a Riesz basis for its closed span which is $X_{1}+X_{2}+X_{3}$.

Proof. For notational simplicity we write the proof for the case of two subsets.

The first conclusion is immediate. Suppose now that we have, for $j=1,2$

$$
Y_{j}=\sum_{k \in I_{j}} a_{j k} x_{k}
$$

We assume $\left\|Y_{1}\right\|=\left\|Y_{2}\right\|=1$ and we need to show $\left|\left\langle Y_{1}, Y_{2}\right\rangle\right| \leq 1-\varepsilon$ for some $\varepsilon>0$. By multiplying $Y_{2}$ by a unimodular constant we can assume that $\left\langle Y_{1}, Y_{2}\right\rangle$ is real and non-positive. We then have

$$
\begin{aligned}
\left\|Y_{1}+Y_{2}\right\|^{2} & =\left\|Y_{1}\right\|^{2}+\left\|Y_{2}\right\|^{2}+2 \operatorname{Re}\left\langle Y_{1}, Y_{2}\right\rangle \\
& =2\left(1+\left\langle Y_{1}, Y_{2}\right\rangle\right) .
\end{aligned}
$$

On the other hand, twice using the fact that we have a Riesz basis we have

$$
\begin{aligned}
\left\|Y_{1}+Y_{2}\right\|^{2} & \geq c \sum_{j=1,2 ; k \in I_{j}}\left|a_{j k}\right|^{2} \\
& =c\left(\sum_{k \in I_{1}}\left|a_{1 k}\right|^{2}+\sum_{k \in I_{2}}\left|a_{2 k}\right|^{2}\right) \\
& \geq c^{\prime}\left(\left\|Y_{1}\right\|^{2}+\left\|Y_{2}\right\|^{2}\right) \\
& \geq 2 c^{\prime}>0 .
\end{aligned}
$$

Combining these two and recalling that $\left\langle Y_{1}, Y_{2}\right\rangle \leq 0$ demonstrates that the subspaces are at a positive angle.

In the other direction we need upper and lower estimates for $\left\|Y_{1}+Y_{2}\right\|$. Using the triangle inequality and the Riesz basis statement for the subsets gives

$$
\begin{aligned}
\left\|Y_{1}+Y_{2}\right\|^{2} & \leq 2\left\|Y_{1}\right\|^{2}+2\left\|Y_{2}\right\|^{2} \\
& \leq 2\left(\sum_{k \in I_{1}}\left|a_{1 k}\right|^{2}+\sum_{k \in I_{2}}\left|a_{2 k}\right|^{2}\right) \\
& =2 \sum_{j=1,2 ; k \in I_{j}}\left|a_{j k}\right|^{2} .
\end{aligned}
$$


In the other direction we have

$$
\begin{aligned}
\left\|Y_{1}+Y_{2}\right\|^{2} & =\left\|Y_{1}\right\|^{2}+\left\|Y_{2}\right\|^{2}+2 \operatorname{Re}\left\langle Y_{1}, Y_{2}\right\rangle \\
& \geq\left\|Y_{1}\right\|^{2}+\left\|Y_{2}\right\|^{2}-2(1-\varepsilon)\left\|Y_{1}\right\|\left\|Y_{2}\right\| \\
& =\varepsilon\left(\left\|Y_{1}\right\|^{2}+\left\|Y_{2}\right\|^{2}\right)+(1-\varepsilon)\left(\left\|Y_{1}\right\|-\left\|Y_{2}\right\|\right)^{2} \\
& \geq \varepsilon\left(\left\|Y_{1}\right\|^{2}+\left\|Y_{2}\right\|^{2}\right) \\
& \geq c \varepsilon 2\left(\sum_{k \in I_{1}}\left|a_{1 k}\right|^{2}+\sum_{k \in I_{2}}\left|a_{2 k}\right|^{2}\right)
\end{aligned}
$$

as required.

The result of interest is

THEOREM 14. Suppose $M$ is as described and satisfies assumptions 1, 2, and 3. Given $B>0$ there is a $B^{\prime}=B^{\prime}(M, B)$ so that if $X \subset M$ is a local interpolating sequence with constant $B$ then $X$ is an interpolating sequence with constant $B^{\prime}$.

The theorem will follow from two lemmas.

Lemma 1. Suppose $X \subset M$ is an interpolating sequence and $y \in M$, then $X \cup\{y\}$ is an interpolating sequence.

Lemma 2. Suppose $X$ is a local interpolating sequence and $Y \subset X$ is an interpolating sequence. Then for any $x \in \partial M$ the set $Y \cup\left(X \cap B\left(x, \varepsilon_{X} / 10\right)\right)$ is an interpolating sequence.

Proof of Lemma 1. If we can find $f$ with $\left.f\right|_{X} \equiv 0$ and $f(y)=1$ then we can solve an interpolation problem on $X \cup\{y\}$ by using functions of the form $g+\lambda f$ where $g$ solves the interpolation problem on $X$ and $\lambda$ is an appropriately chosen scalar. To prove the lemma we now assume there is no such $f$ and obtain a contradiction.

Let $A$ be the closed linear span of $\left\{j_{x_{i}}\right\}_{x_{i} \in X}$. We are assuming that, for $v \in \mathcal{H}$, if $v \perp A$, and thus $v$ vanishes on $X$, then $v(y)=0$, thus $v \perp j_{y}$. Hence $j_{y} \in A^{\perp \perp}$. However by (5.1) $A$ is closed and thus $A^{\perp \perp}=A$ and we conclude that $j_{y} \in A$. Hence by (5.1) we have

$$
j_{y}=\sum \beta_{i} j_{x_{i}}
$$

for a uniquely determined square summable sequence $\left\{\beta_{i}\right\}$. Pick $j$ such that $\beta_{j} \neq 0$ and let $X^{\prime}=X \backslash\left\{x_{j}\right\}$. There must be an $f \in \mathcal{H}$ which vanishes on $X^{\prime}$ and with $f(y) \neq 0$. Otherwise we could argue as we just did and find another representation for $j_{y}$ as in (5.2) however this time with the sum not involving the index $j$. This contradicts the fact that the representation in (5.2) is unique. Thus we have $f$ which vanishes on $X^{\prime}$ and not at $y$. By assumption 1 we can find a multiplier $m$ with $m\left(x_{j}\right)=0$ and $m(y) \neq 0$. Thus the function $m f$ vanishes on $X$ and not at $y$ which is a contradiction.

Proof of Lemma 2. The idea of the proof is straightforward but the details are awkward hence we first describe the idea.

To oversimplify, we will divide the set $Y \cup\left(X \cap B\left(x, \varepsilon_{X} / 10\right)\right)$ into three pieces, $I, N$, and $F$ of points in $X \cap B\left(x, \varepsilon_{X} / 10\right)$, near $X \cap B\left(x, \varepsilon_{X} / 10\right)$ and far from $X \cap$ 
$B\left(x, \varepsilon_{X} / 10\right)$. We denote the closed span of the reproducing kernels corresponding to those three sets by $A_{I}, A_{N}$, and $A_{F}$. We will verify (5.1) for the set of kernels corresponding to $Y \cup\left(X \cap B\left(x, \varepsilon_{X} / 10\right)\right)=I \cup N \cup F$. By the proposition this will follow if we establish that

- That $\left\{j_{i}\right\}_{i \in I}$ is a Riesz basis of $A_{I}$ (i.e. an estimate such as (5.1) holds); similarly for $A_{N}$ and $A_{F}$.

- Each pair $\left(A_{I}, A_{N}\right),\left(A_{I}, A_{F}\right)$, and $\left(A_{N}, A_{F}\right)$ are at a positive angle.

The Riesz basis statement holds for $A_{I}$ because $\left\{x_{i}\right\}_{i \in I}$ a subsequence of $X \cap$ $B\left(x, \varepsilon_{X}\right)$ which, by the assumption on $X$, is an interpolating sequence. The Riesz basis statement holds for the other two spaces because both $\left\{x_{i}\right\}_{i \in N}$ and $\left\{x_{i}\right\}_{i \in F}$ are interpolating sequences by virtue of being subsequences of $Y$. The inclusion of $N$ and $F$ in $Y$ also insures that $A_{N}$ and $A_{F}$ are at a positive angle. We will have $I \cup N \subset X \cap B\left(x, \varepsilon_{X}\right)$ and thus $\left\{x_{i}\right\}_{i \in I \cup N}$ is an interpolating sequence and hence $A_{I}$, and $A_{N}$ will be a positive angle. Finally we need to show that $A_{I}$ and $A_{F}$ are at a positive angle. We select $e \in A_{I}$ and $f \in A_{F}$ and compute $\langle e, f\rangle$ in terms of the the appropriate Riesz bases of normalized kernel functions. We estimate the individual terms in the product using the third assumption, use the CauchySchwartz inequality, and then estimate the result using the second assumption.

To do the actual proof we first break $B\left(x, \varepsilon_{X} / 10\right)$ into smaller pieces. Select $\varepsilon>0$ small, with details to be specified later. Cover $B\left(x, \varepsilon_{X} / 10\right) \cap \partial M$ by a finite set of balls $\left\{B\left(x_{i}, \varepsilon\right)\right\}_{i=1}^{N}$. with centers in $\partial M$. Partition $B\left(x, \varepsilon_{X} / 10\right) \cap X$ into sets $\left\{S_{i}\right\}_{i=1}^{N} \subset B\left(x_{i}, \varepsilon\right)$ together with a residual set $F$. Because $X$ has no interior accumulation points we may take $F$ to be finite. At the end of the proof we use the first lemma repeatedly to adjoin the points of $F$ to our interpolating sequence. We won't speak of $F$ again.

Let $Y_{0}=Y \backslash\left(Y \cap\left(\cup_{i=1}^{M} S_{i}\right)\right.$ and for $i=1,2, \ldots, M$ let $Y_{i}=Y_{i-1} \cup S_{i}$. We will establish inductively that each $Y_{i}$ is an interpolating sequence. When the process stops at $i=M$ the proof will be complete. $Y_{0}$ is a subsequence of the interpolating sequence $Y$ and hence is an interpolating sequence. We now suppose $Y_{i-1}$ is an interpolating sequence. We divide $Y_{i-1} \cup S_{i}$ into three subsets, $I=S_{i}$,

$$
N=Y_{i-1} \cap B\left(x, \varepsilon_{X} / 10\right)
$$

and $F=Y_{i-1} \backslash(I \cup N)$. Let the corresponding spans by denoted $A_{I}, A_{N}$, and $A_{F}$. The easily modification of earlier discussion shows the set $\left\{j_{i}\right\}_{i \in I}$ is a Riesz basis of $A_{I}$ and similarly for $A_{N}$ and $A_{F}$. That discussion also indicates why the pairs $\left(A_{I}, A_{N}\right)$ and also $\left(A_{F}, A_{N}\right)$ are at positive angle.

We now need to show $A_{I}$ and $A_{F}$ are at a positive angle. Pick $a_{I} \in A_{I}$ and $b_{F} \in A_{F}$ and write these functions in terms of the normalized reproducing kernels for points in the appropriate sequences.

$$
a_{I}=\sum_{x_{j} \in I} \alpha_{j} j_{x_{j}} ; b_{F}=\sum_{x_{l} \in F} \beta_{l} j_{x_{l}} .
$$


We compute

$$
\begin{aligned}
\left\langle a_{I}, b_{F}\right\rangle & =\left\langle\sum_{x_{j} \in I} \alpha_{j} j_{x_{j}}, \sum_{x_{l} \in F} \beta_{l} j_{x_{l}}\right\rangle \\
& =\sum_{j, l} \alpha_{j} \overline{\beta_{l}}\left\langle j_{x_{j}}, j_{x_{l}}\right\rangle \\
& =\sum_{j, l} \alpha_{j} \overline{\beta_{l}}\left\|J_{x_{l}}\right\|^{-1} j_{x_{j}}\left(x_{l}\right)
\end{aligned}
$$

So

$$
\left|\left\langle a_{I}, b_{F}\right\rangle\right| \leq \sum_{j, l}\left|\alpha_{j}\right| \overline{\left|\beta_{l}\right|}\left\|J_{x_{l}}\right\|^{-1} j_{x_{j}}\left(x_{l}\right)
$$

We now use the third assumption to dominate the numerator by a constant $C$ and continue by factoring the sum and then using the Cauchy-Schwartz inequality

$$
\begin{aligned}
\left|\left\langle a_{I}, b_{F}\right\rangle\right| & \leq \sum_{j, l}\left|\alpha_{j}\right| \overline{\left|\beta_{l}\right|}\left\|J_{x_{l}}\right\|^{-1} j_{x_{j}}\left(x_{l}\right) \\
& \leq C \sum_{j, l}\left|\alpha_{j}\right| \overline{\left|\beta_{l}\right|}\left\|J_{x_{j}}\right\|^{-1}\left\|J_{x_{l}}\right\|^{-1} \\
& \leq C\left(\sum_{j}\left|\alpha_{j}\right| \|\left. J_{x_{j}}\right|^{-1}\right)\left(\sum_{l} \overline{\left|\beta_{l}\right|}\left\|J_{x_{l}}\right\|^{-1}\right) \\
& \leq C\left(\sum_{j}\left|\alpha_{j}\right|^{2}\right)^{1 / 2}\left(\sum_{j}\left\|J_{x_{j}}\right\|^{-2}\right)^{1 / 2}\left(\sum_{l}{\overline{\left|\beta_{l}\right|}}^{2}\right)^{1 / 2}\left(\sum_{l}\left\|J_{x_{l}}\right\|^{-2}\right)^{1 / 2}
\end{aligned}
$$

As we mentioned earlier, the fact that $X$ is a local interpolating sequence insures that the associated Carleson measure is finite. Thus we continue with

$$
\left|\left\langle a_{I}, b_{F}\right\rangle\right| \leq C C^{\prime}\left(\sum_{j}\left|\alpha_{j}\right|^{2}\right)^{1 / 2}\left(\sum_{x_{j} \in I}\left\|J_{x_{j}}\right\|^{-2}\right)^{1 / 2}\left(\sum_{l}{\overline{\mid \beta_{l}}}^{2}\right)^{1 / 2}
$$

We now use that

$$
\left(\sum_{j}\left|\alpha_{j}\right|^{2}\right)^{1 / 2} \sim\left\|a_{I}\right\|
$$

with constants determined by the fact that all the points of $I$ are in $B\left(x, \varepsilon_{X} / 5\right)$ which, because $X$ is a local interpolating sequence, is an interpolating sequence. In particular these constants don't depend on our induction index $i$. Similarly

$$
\left(\sum_{l}{\overline{\left|\beta_{l}\right|}}^{2}\right)^{1 / 2} \sim\left\|b_{F}\right\|
$$


with constants that only depend on the interpolation data of the set $Y$, and thus are again independent of the index $i$. Thus

$$
\begin{aligned}
\left|\left\langle a_{I}, b_{F}\right\rangle\right| & \leq C^{\prime \prime}\left(\sum_{x_{j} \in I}\left\|J_{x_{j}}\right\|^{-2}\right)^{1 / 2}\left\|a_{I}\right\|\left\|b_{F}\right\| \\
& =C^{\prime \prime} \mu_{I}\left(B\left(x_{i}, \varepsilon\right)\right)^{1 / 2}\left\|a_{I}\right\|\left\|b_{F}\right\| \\
& \leq C^{\prime \prime} \gamma(\varepsilon)^{1 / 2}\left\|a_{I}\right\|\left\|b_{F}\right\| .
\end{aligned}
$$

The constant $C^{\prime \prime}$ only depends on data that was available before the start of the proof. Hence at the start we can choose $\varepsilon$ so that $C^{\prime \prime} \gamma(\varepsilon)^{1 / 2}<1$ which completes the proof.

Proof of Theorem 14. Write $X$ as a disjoint union $\left\{X_{i}\right\}_{i=1}^{K}$ with each $X_{i}$ contained in a ball centered on the boundary of radius $\varepsilon_{X} / 20$, together with a residual finite set $F$. Apply the second lemma inductively to show that for $j=$ $1, \ldots, K$ the set $\cup_{i=1}^{j} X_{i}$ is an interpolating sequence. Then use the first lemma to adjoin $F$.

Remark 2. Proposition 9 and Remark 10 of [ARS3] give a description of the Carleson measures which are supported on complex tangential sets in terms of a tree condition.

REMARK 3. There are other different conditions that imply that $Z$ is a local interpolating sequence. For instance if $n>1$ is $Z$ sits in the disk $\{(z, 0, \ldots, 0):|z|<1\}$. In that case by appeal to the classical Hardy space theory, Theorem 5 above, if $Z$ satisfies (CM) and (SEP) it is an interpolating sequence and hence, a fortiori, a local interpolating sequence. Note that in this case the fact that such a sequence is interpolating cannot be obtained from Boe's theorem because Condition B is not satisfied for the Hardy space kernels.

\section{References}

[AM] J. Agler and J. E. $\mathrm{M}^{c}$ Carthy, Pick interpolation and Hilbert function spaces. AMS 2002.

[AC] P. Ahern, W. Cohn, Exceptional sets for Hardy Sobolev functions, $p>1$, Indiana Univ. Math. J. 38 (1989), no. 2, 417-453.

[ARS1] N. Arcozzi, R. Rochberg and E. Sawyer, Carleson measures for analytic Besov spaces, Rev. Mat. Iberoamericana 18 (2002), 443-510.

[ARS2] N. Arcozzi, R. Rochberg and E. Sawyer, Carleson measures and interpolating sequences for Besov spaces on complex balls, Memoirs Amer. Math. Soc. 859, 2006.

[ARS3] N. Arcozzi, R. Rochberg and E. Sawyer, Carleson Measures for the DruryArveson Hardy space and other Besov-Sobolev Spaces on Complex Balls, available at http://www.math.mcmaster.ca/ ${ }^{\text {s sawyer. }}$

[A] W. B. Arveson, Subalgebras of $C^{*}$-algebras III: multivariable operator theory, Acta Math. 181 (1998), 159-228.

[BCR] C, Berg, J, Christensen, P. Ressel, Harmonic analysis on semigroups. Theory of positive definite and related functions. Graduate Texts in Mathematics, 100. Springer-Verlag, New York, 1984.

[B1] B. Böe, Interpolating sequences for Besov spaces. J. Funct. Anal. 192 (2002), no. 2, 319-341.

[B2] B. Boe, An interpolation theorem for Hilbert spaces with Nevanlinna-Pick kernel, Proc. Amer. Math. Soc. 210 (2003).

[Ch] Z. Chen, Characterizations of Arveson's Hardy space, Complex Variables 48 (2003), 453465. 
[N] N. Nikolski, . Operators, functions, and systems: an easy reading. Vol. 1. Hardy, Hankel, and Toeplitz. Translated from the French by Andreas Hartmann. Mathematical Surveys and Monographs, 92. American Mathematical Society, Providence, RI, 2002.

[P] S. C. Power, Hankel operators on Hilbert space. Hankel operators on Hilbert space. Research Notes in Mathematics, 64. Pitman (Advanced Publishing Program), Boston, Mass.London, 1982.

[PV] M. Putinar, F, Vasilescu, Solving moment problems by dimensional extension. Ann. of Math. (2) 149 (1999), no. 3, 1087-1107.

[Z] K. Zhu, Operator theory in function spaces. Monographs and Textbooks in Pure and Applied Mathematics, 139. Marcel Dekker, Inc., New York, 1990. 